\title{
Crescimento, produção e qualidade de frutos de atemoieira 'Gefner' submetida a diferentes intensidades de poda
}

\author{
Growth, yield and fruit quality of atemoya trees 'Gefner' under different pruning intensities
}

\author{
Virgílio Jamir Gonçalves Mota Filho ${ }^{\mathrm{I}^{*}}$ Marlon Cristian Toledo Pereira ${ }^{\mathrm{I}}$ Silvia Nietsche ${ }^{\mathrm{I}}$ \\ Victor Martins Maia ${ }^{\mathrm{I}}$ Marcos Gleidson Pereira dos Santos ${ }^{\mathrm{II}}$ Thiago Prates Fernandes ${ }^{\mathrm{I}}$
}

\section{RESUMO}

Incrementos na produtividade e na qualidade dos frutos de atemoieira, principalmente no período de entressafra, são prioridades dos produtores. O presente trabalho teve como objetivo avaliar o crescimento e a produção da atemoieira 'Gefner' submetida a diferentes intensidades de poda. O delineamento experimental foi o de blocos casualizados com cinco tratamentos, quatro repetições e uma planta por parcela. Os tratamentos utilizados foram ramos podados com 10, 20, 30, 40 e $50 \mathrm{~cm}$ de comprimento. Foram avaliados o número de flores, comprimento e diâmetro dos ramos brotados, diâmetro da copa e altura da planta, número de frutos na pré-colheita e colheita, peso total de frutos, peso médio de frutos, diâmetro dos frutos, comprimento dos frutos, peso da casca dos frutos, peso de polpa e semente dos frutos, teor de sólidos solúveis, acidez titulável e pH. As características avaliadas foram submetidas à análise de variância, tendo os efeitos dos diferentes comprimentos dos ramos podados testados e ajustados em equações de regressão. Não foram observados efeitos significativos sobre as características altura de plantas, diâmetro da copa, peso total de frutos, diâmetro dos frutos, comprimento dos frutos, peso da casca dos frutos, peso de polpa e semente dos frutos, teor de sólidos solúveis, acidez titulável e $\mathrm{pH}$. Entretanto, foi observada redução linear do número de flores, comprimento e diâmetro dos ramos brotados, à medida que aumentou a intensidade de poda.

Palavras-chave: Annona cherimolia x Annona squamosa, comprimento do ramo, peso de frutos.

\section{ABSTRACT}

Improvements in yield and fruit quality in atemoya, mainly during the period of out of season are priorities for growers. The purpose of this study was to evaluate the growth and yield of atemoya 'Gefner' under different pruning intensities. The experimental design was randomized blocks, with five treatments, four replications and one plant per block. The treatments were pruned to 10, 20, 30, 40 and 50cm in length. Number of flowers, length and diameter of branches sprouted, crown diameter, plant height, number of fruits in pre-harvest and harvest periods, total weight of fruits, fruit weight, fruit diameter, fruit length, peel, pulp and seed weight, soluble solids, titratable acidity and $\mathrm{pH}$ were evaluated. The characteristics were submitted to analysis of variance, and the effects of different lengths of branches prunedtested and adjusted in the regression equation. No significant influence were observed fordiameter of the crown, plant height, number of fruit in pre-harvest and harvest periods, totalweight of fruits, fruit weight, fruit diameter, fruit length, peel, pulp and seed weight, solublesolids, titratable acidity and $\mathrm{pH}$. However, as the increased pruning intensities showed a linear reduction in the number of flowers, length and diameter of branches sprouted in atemoya tree 'Gefner'.

Key words: Annona cherimolia x Annona squamosa, branch length, fruit weight.

\section{INTRODUÇÃO}

A poda é uma das mais antigas práticas hortícolas adotadas em fruteiras de clima temperado. Entretanto, para espécies tropicais e subtropicais, poucos estudos têm sido conduzidos sobre o efeito de diferentes intensidades de podas quanto à penetração de luz, pegamento de frutos e produção (SHARMA \& SINGH, 2006).

A atemóia (cherimoia Annona cherimola MILL. X pinha Annona squamosa L.) é uma planta semi-decídua de origem subtropical que, em geral, perde suas folhas progressivamente na primavera (OLESEN \& MULDOON, 2009). O

'Universidade Estadual de Montes Claros (UNIMONTES), Rua Reinaldo Viana, 2630, 39440-000, Janaúba, MG, Brasil. E-mail: virgiliojamir@yahoo.com.br.*Autor para correspondência.

IDDepartamento de Fitotecnia, Universidade Federal de Viçosa (UFV), Viçosa, MG, Brasil. 
alongamento dos brotos vegetativos é contínuo, iniciando-se na primavera até o próximo inverno. Em geral, o desenvolvimento dos ramos é linear e até o final da estação podem atingir comprimento de $3 \mathrm{~m}$ ou mais (OLESEN \& MULDOON, 2009). O florescimento ocorre restritamente no estádio inicial de desenvolvimento dos brotos e opostos às folhas (GEORGE \& NISSEN, 1991).

Na Austrália, a poda em plantas de atemoieira deve ser realizada no final do inverno ou no início da primavera, evitando-se podas severas com comprimento de ramos inferiores a 20cm (GEORGE et al., 2001). Nos perímetros irrigados do semiárido brasileiro, recomenda-se a poda preferencialmente entre os meses de julho a setembro, embora possa ser realizada em qualquer época do ano, tendo em vista a elevada temperatura e o uso da irrigação (PEREIRA et al., 2011).

A intensidade da poda em plantas frutíferas pode afetar a brotação, florescimento e consequente produção e qualidade dos frutos. DIAS et al. (2003), testando poda de ramos da pinheira com diferentes diâmetros, observaram que ramos com maior diâmetro imprimem maior vigor às brotações e flores, mas não afetam a velocidade de crescimento e as características físico-químicas dos frutos. GEORGE et al. (2001) podaram seis ramos com folhas por planta de atemóia durante a primavera e verão e obtiveram incrementos significativos no tamanho dos frutos, sendo maior quanto mais cedo a poda foi realizada. No entanto, não foram encontrados na literatura trabalhos referentes à intensidade de poda no cultivo da atemoieira.

Dessa maneira, o objetivo do presente trabalho foi avaliar o crescimento e a produção da atemoieira 'Gefner', submetida a diferentes intensidades de poda nas condições irrigadas do Norte de Minas Gerais.

\section{MATERIAL E MÉTODOS}

O experimento foi implantado em área cultivada com 12 ha de atemoieira 'Gefner' com nove anos de idade, e com espaçamento 4x2,5m, localizada no município de Matias Cardoso, Vale do São Francisco, região Norte de Minas Gerais. A irrigação utilizada foi de microaspersão e os tratos culturais e fitossanitários foram realizados de acordo com as recomendações descritas por PINTO et al. (2005).

O experimento foi implantado no dia 29 de maio de 2008, quando foram feitas as podas em 20 plantas de atemoia. As plantas selecionadas foram disponibilizadas na mesma linha de plantio, observando uniformidade, vigor e sanidade. As plantas foram podadas por completo, com tesoura de poda, deixando os ramos com comprimentos estabelecidos conforme os tratamentos descritos a seguir, seguida da desfolha manual. O delineamento experimental foi o de blocos casualizados, com cinco tratamentos, com quatro repetições e uma planta por parcela. Os tratamentos utilizados foram ramos podados com 10, 20, 30, 40 e 50cm de comprimento.

Para a avaliação das características, foram amostrados dois ramos podados em cada quadrante, no terço médio da planta, com espessura média de 11mm. Esses ramos foram marcados com fitas de lã coloridas. Em cada ramo podado, foi escolhido aleatoriamente um ramo brotado, o qual foi devidamente identificado para as avaliações.

A polinização artificial foi realizada entre os dias 29 e 31 de julho, entre 7-9h da manhã. Foram utilizados grãos de pólen de pinha e atemoia em proporções iguais, adicionando-se $30 \%$ de talco à mistura. A polinização artificial foi realizada por meio de pincel número dois. $\mathrm{O}$ desbaste de frutos foi feito no dia 16 de setembro de 2008, 109 dias após a poda, que consistiu na retirada de frutos mal formados e doentes, de forma uniforme em todas as plantas.

Sessenta dias após a poda de produção, foi realizada a contagem do número de flores nos ramos brotados previamente identificados e, em torno de 180 dias, foram obtidos o diâmetro e comprimento desses mesmos ramos. A medida do diâmetro foi obtida por meio de um paquímetro e o comprimento através de uma fita métrica. No dia 16 de setembro de 2008, logo após o desbaste dos frutos, foi realizada a contagem de todos os frutos presentes em cada planta.

No dia 9 de dezembro de 2008, cerca de seis meses após a poda de produção, foram efetuadas as medidas do diâmetro das copas das plantas no sentido da linha e da rua, além da altura, com auxílio de uma trena.

A colheita dos frutos foi realizada entre os dias 08 a 21 de janeiro de 2009, retirando-se os frutos no ponto de maturação fisiológica, que é a passagem de cor da casca de verde-claro-brilhante para verde-amarelado-pálido. Os frutos colhidos foram acondicionados em caixas de papelão devidamente identificadas e enviadas ao laboratório de fisiologia pós-colheita de frutos da Universidade Estadual de Montes Claros, onde foi obtido o peso total dos frutos por planta, comprimento e diâmetro dos frutos. Ao atingirem o ponto de consumo, que ocorreu cinco dias após a colheita, avaliou-se o peso dos frutos, peso de casca e peso de polpa com semente. Também se avaliou o teor de sólidos solúveis, retirando- 
se amostra da polpa da região central do fruto, utilizando-se refratômetro digital Reichert. Para a medição da acidez titulável e $\mathrm{pH}$, retiraram-se $10 \mathrm{~g}$ de polpa da região central do fruto, triturando-se e homogeneizando-se com $90 \mathrm{~mL}$ de água destilada, utilizando-se liquidificador industrial. Em seguida, a acidez titulável foi medida em eq mg ${ }^{-1}$ de ácido cítrico, utilizando-se o método de titulometria, enquanto o $\mathrm{pH}$ foi medido por pHmetro digital da marca Gehaka, modelo PG 1800 (AOAC, 1990).

As características avaliadas foram submetidas à análise de variância, tendo os efeitos dos diferentes comprimentos de ramos podados testados e ajustados em equação de regressão. As análises estatísticas foram efetuadas com o auxílio do Programa de Análises Estatísticas e Planejamento de Experimentos da Universidade Federal de Lavras (SISVAR-UFLA).

\section{RESULTADOS E DISCUSSÃO}

Com o aumento no comprimento de ramos podados, foi observada redução linear no número de flores $(\mathrm{P}<0,05)$, verificada nos ramos brotados aos dois meses após a poda (Figura 1). Esse comportamento pode ser explicado devido ao menor vigor dos ramos brotados em ramos podados mais longos (Figuras 2 e 3). Com o menor comprimento dos ramos brotados, possivelmente, ocorreu menor quantidade de gemas florais. DIAS et al. (2004) encontraram resultados contrários trabalhando com podas de 5,
10, 15, 20, 25 e $30 \mathrm{~cm}$ de comprimento em pinha na região de Anagé-BA. Esses autores observaram incremento no número de botões florais, emitidos aos 28 dias após a poda, com o aumento do comprimento do ramo podado.

Segundo PAULL \& DUARTE (2011), um dos fatores que induz o florescimento é a relação carbono/nitrogênio $(\mathrm{C} / \mathrm{N})$. A relação $\mathrm{C} / \mathrm{N}$ fica mais baixa quando se faz um encurtamento mais drástico dos ramos, terminando nos tecidos próximos à base, ocasionando um crescimento mais vigoroso e menos frutífero. No presente experimento, possivelmente a relação C/N dos ramos de atemoia 'Gefner' com encurtamento mais drástico pode não ter alterado suficientemente para reduzir o florescimento. O tratamento com $20 \mathrm{~cm}$ de comprimento atingiu média de 1,29 flores por ramo brotado (Figura 1). Estudos conduzidos por SOLER \& CUEVAS (2009) no sudeste da Espanha, em plantas de cherimoia, cultivar Fino de Jete, obtiveram médias de 1,08 flores por ramo podado. Na região Norte de Minas Gerais, muitos produtores têm utilizado a poda de produção com encurtamento de ramos a $20 \mathrm{~cm}$ de comprimento, prática recomendada por GEORGE et al. (2001).

Observou-se redução linear do comprimento e diâmetro dos ramos brotados com o aumento do comprimento dos ramos podados $(\mathrm{P}<0,05)$ (Figuras 2 e 3). Portanto, ramos com poda drástica ou mais curta dos ramos apresentaram maior vigor nos ramos brotados, resposta esta aos seis meses após a poda. Esses resultados estão de acordo

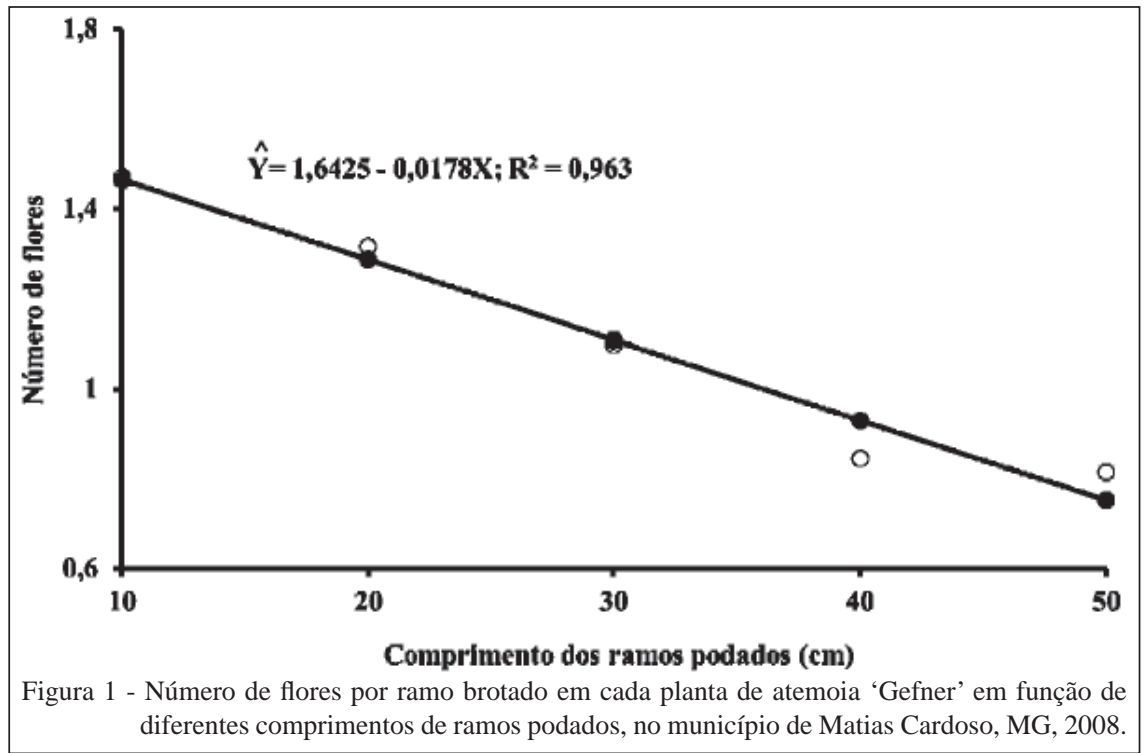

Ciência Rural, v.43, n.11, nov, 2013. 


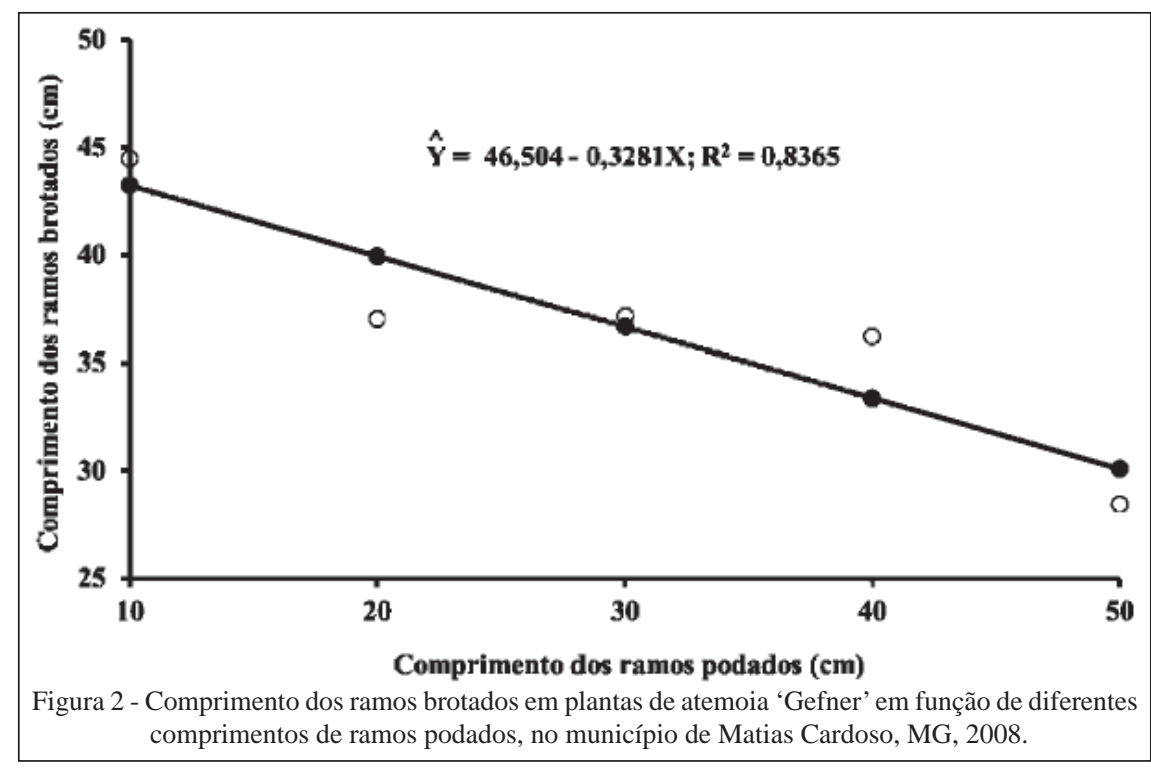

com os princípios que regem a poda, ou seja, a maior severidade da poda com o encurtamento dos ramos proporciona maior vigor dos ramos brotados (WADE \& WESTERFIELD, 2009). DIAS et al. (2004), trabalhando com ramos de pinheira podados com 5 , $10,15,20$ e $25 \mathrm{~cm}$ de comprimento, verificaram que os ramos podados com os menores comprimentos tendem a aumentar o vigor das brotações, resultados semelhantes ao presente trabalho.

Os diferentes comprimentos de ramos podados não influenciaram significativamente nas características da altura da planta, diâmetro da copa da planta no sentido da linha de plantio e da rua, com médias de 2,92m, 3,00m e 4,10m, respectivamente. Conforme PINTO et al. (2005), aconselham-se plantas com altura máxima de 3,0m, o que facilita as práticas culturais como polinização, desbaste, ensacamento de frutos, colheita, dentre outros.

Também não foram verificadas diferenças significativas $(\mathrm{P}<0,05)$ entre os diferentes comprimentos de ramos podados para as características número de frutos na pré-colheita por planta, número de frutos na colheita por planta, peso total de frutos por planta, peso médio de frutos por planta, diâmetro dos frutos, comprimento dos frutos, peso da casca, peso de polpa e semente.

O número médio de frutos por planta foi de 63, valor que está próximo à recomendação técnica de deixar de 60 a 80 frutos após o desbaste, embora dependa do porte da planta (PINTO et al., 2005).
SHARMA \& SINGH (2006), estudando intensidade de poda em mangueira 'Amrapali', observaram maior número de panículas com frutos quando efetuaram poda moderada dos ponteiros dos ramos, ou seja, com remoção de $20 \mathrm{~cm}$ da parte a partir do ápice do ramo.

A média de frutos colhidos foi de 44 e o peso médio de cada fruto foi de $214 \mathrm{~g}$, valores inferiores aos obtidos por OLESEN \& MULDOON (2009) que obtiveram médias de 81 frutos e $353 g$ por fruto de atemoia 'AfricanPride'. Esses resultados podem ter sido influenciados pelas diferenças entre as cultivares e as condições edafoclimáticas nas quais os experimentos foram conduzidos. Os frutos de atemoieira 'Gefner' apresentaram, em média, 72mm e 82mm de diâmetro e comprimento, respectivamente, demonstrando frutos de adequado padrão comercial.

O peso total de frutos por planta, peso da casca e peso de polpa com semente apresentaram médias de 9,60kg, 41,16g e 215,89g, respectivamente. A ausência de diferenças significativas entre as características produtivas em função dos diferentes comprimentos de ramos podados pode ser explicada por um possível equilíbrio de frutificação na planta. Possivelmente, os ramos podados em maior comprimento tenham emitido mais brotações, por possuir mais gemas ao longo desse ramo, e, mesmo que essas brotações tenham apresentado individualmente menor vigor e menor quantidade de flores, quando considerados os dados de número de frutos e produção da planta inteira, houve um equilíbrio da quantidade 


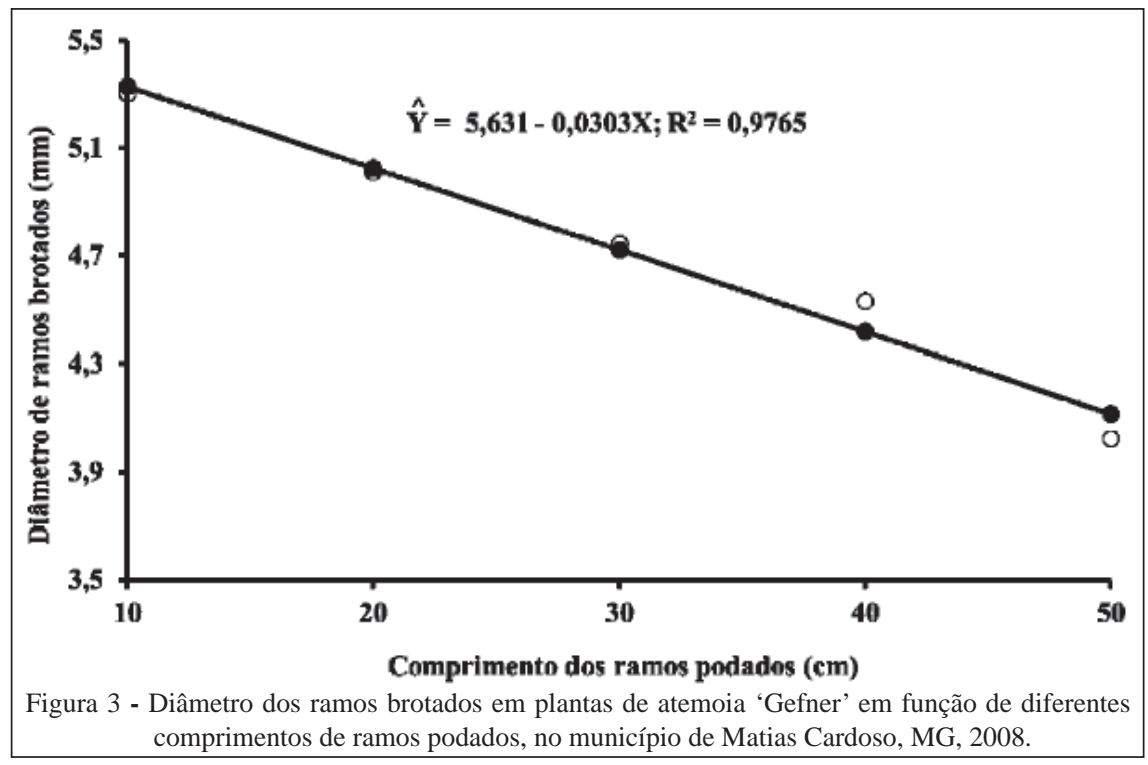

e qualidade de frutos, quando comparado às plantas que possuíam ramos podados mais curtos.

As características químicas teor de sólidos solúveis, acidez titulável e $\mathrm{pH}$ não foram afetadas significativamente $(\mathrm{P}<0,05)$ pelos diferentes comprimentos de ramos podados. Com relação ao teor de sólidos solúveis, acidez titulável e $\mathrm{pH}$, a polpa dos frutos apresentou médias de 25,2 Brix, 0,72\% de ácido cítrico e 4,86, respectivamente. Resultados similares também foram obtidos por LEMOS et al. (2003) e MEDEIROS et al. (2009), demonstrando a baixa variação dessas características, no entanto, com excelente qualidade para o consumo. A ausência de efeitos significativos dos diferentes comprimentos de ramos podados nessas características pode ser explicada também pelo equilíbrio da quantidade de frutos em cada planta, possivelmente proporcionando distribuição de fotoassimilados em quantidade uniforme aos frutos de atemoieira 'Gefner'.

\section{CONCLUSÃO}

O número de flores, comprimento e diâmetro de ramos brotados são reduzidos linearmente à medida que os ramos da atemoieira 'Gefner' podados são deixados maiores, ou seja, de 10 para 50cm de comprimento.

Não há influência do comprimento dos ramos podados sobre altura e diâmetro das copas das plantas, produção e características físico-químicas dos frutos da atemoieira 'Gefner'.

\section{REFERÊNCIAS}

AOAC (ASSOCIATION OF OFFICIAL ANALITICAL CHEMISTS). Official methods of analisys of the Association of Official Analitical Chemists. 15.ed. Washington, 1990. 842p.

DIAS, N.O. et al. Influência da poda de produção em ramos de diferentes diâmetros no desenvolvimento vegetativo e reprodutivo da pinheira (Annona squamosa L.). Revista Brasileira de Fruticultura, Jaboticabal, v.25, n.1, p.100103, 2003. Disponível em: <http://dx.doi.org/10.1590/S010029452003000100029>. Acesso em: 16 dez. 2012. doi: 10.1590/ S0100-29452003000100029.

DIAS, N.O. et al. Desempenho vegetativo e reprodutivo da pinheira (Annona squamosa L.) em função de diferentes comprimentos de ramos podados. Revista Brasileira de Fruticultura, Jaboticabal, v.26, n.3, p.389-391, 2004. Disponível em: <http://dx.doi. org/10.1590/S0100-29452004000300004>. Acesso em: $16 \mathrm{dez}$. 2012. doi: $10.1590 / \mathrm{S} 0100-29452004000300004$.

GEORGE, A.P.; NISSEN, R.J. Annona cherimola Miller Annona squamosa L. A. cherimola x A. squamosa. In: VERHEIJ, E.W.M; CORONEL, R.E. (Eds.). Prosea - plant resource of SouthEast Asia. Edible fruits and nuts. Wageningen: Pudoc Scientific Publishers, 1991. V.2, p.71-75.

GEORGE, A.P. et al. Effects of pruning, girdling and paclobutrazol on shoot growth, yield and quality of atemoya (Annona spp. hybrid) cv. 'African Pride' in subtropical Australia. Thai Journal Agricultural Science, Tailandia, v.34, p.205-215, 2001.

LEMOS, E.P. et al. Efeito da desfolha de ramos sobre a indução brotos e flores em atemóia (Anonna cherimola Mill x Anonna squamosa L). Revista Brasileira de Fruticultura, Jaboticabal, v.25， n.1, p.170-171, 2003. Disponível em: <http://dx.doi. org/10.1590/S0100-29452003000100046>. Acesso em: $16 \mathrm{dez}$. 2012. doi: 10.1590/S0100-29452003000100046.

MEDEIROS, P.V.Q. et al. Physical-chemical characterization of atemoya fruit in different maturation stages. Revista Caatinga, Mossoró, v.22, n.2, p.87-90, 2009. 
OLESEN, T.; MULDOON, S.J. Branch development in custard apple (cherimoya Annona cherimola Miller x sugar apple A. squamosa L.) in relation to tip-pruning and flowering, including effects on production. Trees, Vancouver, v.23, p.855-862, 2009. Disponível em: <http://link.springer.com/article/10.1007/s00468009-0327-y/fulltext.html>. Acesso em: 16 dez. 2012. doi: 10.1007/s00468-009-0327-y.

PAULL, E.R.; DUARTE, O. Tropical fruits. 2.ed. Oxford: CAB International, 2011. V.1, 400p. (Crop production science in horticulturae series, 20).

PEREIRA, M.C.T. et al. Anonáceas: pinha, atemoia e graviola. Informe Agropecuário, Belo Horizonte, v.32, n.264, p.26-34, 2011.

PINTO, A.C.Q. et al. Annona species. Southampton, UK: University of Southampton-International Centre for Underutilised
Crops, 2005. 268p. (University of Southampton. Fruits for the Future, 5).

SHARMA, R.R.; SINGH, R. Pruning intensity modifies canopy microclimate, and influences sex ratio, malformation incidence and development of fruited panicles in 'Amrapali' mango (Mangifera indica L.). Scientia Horticulturae, New Delhi, v.109, p.118122, 2006. Disponível em: <http://dx.doi.org/10.1016/j. scienta.2006.03.010>. Acesso em: $16 \mathrm{dez}$. 2012. doi: 10.1016/j. scienta.2006.03.010.

SOLER, L.; CUEVAS, J. Early flower initiation allows ample manipulation of flowering time in cherimoya (Annona cherimola Mill.). Scientia Horticulturae, Almería, v.121, p.327-332, 2009. Disponível em: <http://dx.doi.org/10.1016/j.scienta.2009.02.005>. Acesso em: 16 dez. 2012. doi: 10.1016/j.scienta.2009.02.005.

WADE, G.L.; WESTERFIELD, R.R. Basic principles of pruning woody plants. Athens: University of Georgia, 2009. 6p. (Bulletin 949). 